\title{
Co-morbidity and patterns of care in stimulant-treated children with ADHD in the Netherlands
}

\author{
Adrianne Faber · Luuk J. Kalverdijk • Lolkje T. W. de Jong-van den Berg • \\ Jacqueline G. Hugtenburg • Ruud B. Minderaa • \\ Hilde Tobi
}

Received: 5 November 2007 / Accepted: 21 October 2009/Published online: 6 November 2009

(C) The Author(s) 2009. This article is published with open access at Springerlink.com

\begin{abstract}
This study aimed at investigating the use of psychosocial interventions and psychotropic co-medication among stimulant-treated children with attention-deficit hyperactivity disorder (ADHD) in relation to the presence of psychiatric co-morbidity. Stimulant users younger than 16 years were identified in 115 pharmacies and a questionnaire was sent to their stimulant prescribing physician. Of 773 questionnaires sent out, 556 were returned and were suitable for analysis (72\%). The results are based on 510 questionnaires concerning stimulant-treated children for whom a diagnosis of ADHD was reported. Of the 510 children diagnosed with ADHD, 31\% had also received
\end{abstract}

During the study, Adrianne Faber and Hilde Tobi were working at the Department of Social Pharmacy, Pharmacoepidemiology and Pharmacotherapy, GUIDE Graduate School for Drug Exploration, Groningen, The Netherlands.

\footnotetext{
A. Faber $(\bowtie)$

SIR Institute for Pharmacy Practice and Policy,

Theda Mansholtstraat 5b, $2331 \mathrm{JE}$ Leiden, The Netherlands

e-mail: adrianne.faber@chello.nl
}

L. J. Kalverdijk · R. B. Minderaa

University Centre for Child and Adolescent Psychiatry,

Groningen, The Netherlands

L. T. W. de Jong-van den Berg

Department of Social Pharmacy, Pharmacoepidemiology

and Pharmacotherapy, GUIDE Graduate School for Drug

Exploration, Groningen, The Netherlands

J. G. Hugtenburg

Department of Clinical Pharmacology and Pharmacy,

VU University Medical Center, Amsterdam, The Netherlands

H. Tobi

Social Sciences, Research Methodology Group,

Wageningen University Research, Wageningen,

The Netherlands one or more other psychiatric diagnoses, mainly pervasive developmental disorder or oppositional defiant disorder/ conduct disorder. We found an association between the presence of co-morbidity and the use of psychosocial interventions for the child $(P<0.001)$ and the parents $(P<0.001)$. In the ADHD-only group, $26 \%$ did not receive any form of additional interventions, while psychosocial interventions varied from 8 to $18 \%$ in children with ADHD and psychiatric co-morbidity. The presence of diagnostic co-morbidity was also associated with the use of psychotropic co-medication (overall, $P=0.012$ ) and antipsychotics $(P<0.001)$. Stimulant-treated youths with ADHD and psychiatric co-morbidity received more psychosocial interventions and psychotropic co-medication than children with ADHD-only. The type of psychosocial interventions and psychotropic co-medication received by the children and their parents, depended on the specific co-morbid psychiatric disorder being present.

Keywords Stimulants - Co-morbidity ·

Attention deficit hyperactivity disorder - Co-medication .

Multimodal treatment

\begin{tabular}{|c|c|}
\hline \multicolumn{2}{|c|}{ Abbreviations } \\
\hline ODD & Oppositional defiant disorder \\
\hline CD & Conduct disorder \\
\hline DSM & $\begin{array}{l}\text { Diagnostic and statistical manual of mental } \\
\text { disorder }\end{array}$ \\
\hline MTA & $\begin{array}{l}\text { Multimodal treatment study of children with } \\
\text { ADHD }\end{array}$ \\
\hline PDD & Pervasive developmental disorder \\
\hline PDD-NOS & $\begin{array}{l}\text { Pervasive developmental disorder-not } \\
\text { otherwise specified }\end{array}$ \\
\hline GP & General practitioner \\
\hline
\end{tabular}




\section{Introduction}

Stimulants are first-line drugs in the treatment of attentiondeficit hyperactivity disorder (ADHD) [2, 3]. The rapid increase of stimulant use among children in the 90s [19, 20, $22,27,28,33]$ has raised global concern about the use of stimulants $[1,12,19,26,27,33]$. One of the concerns is the application of stimulant drugs without other forms of treatment, especially since there has been increasing awareness that many children with ADHD also have other co-morbid psychiatric disorders that warrant special treatment consideration [10, 14, 29]. Suggested treatment options depend on the primary diagnosis and the presence of co-morbidity. For instance, for children with ADHD and co-morbid oppositional defiant or conduct disorder (ODD/ $\mathrm{CD})$ symptoms, experts recommend a combination treatment of psychostimulant medication and psychosocial intervention such as parent management training and cognitive behavioural therapy [14]. On the other hand, children with impaired motor skills may benefit from psychostimulant medication combined with treatment by an occupational or physiotherapist [10]. Until now, treatment of ADHD with co-existing psychiatric disorders is guided more by physicians' experience than clinical trials, and more trials should be undertaken [29].

The few studies examining treatment patterns among children with ADHD and psychiatric co-morbidity originated from the USA. Two of these studies focused primarily on psychotropic pharmacotherapy patterns. Among a cohort children aged 5-12 years, Boles et al. found that children with ADHD and psychiatric co-morbidity were more likely to use a combination of psychostimulant and other psychotropic medications than children with uncomplicated ADHD (28 versus 12\%) [4]. A comparable result was found by Radigan et al. among a group of 3-19 year old children [21]. Children with ADHD and two or more co-morbid psychiatric disorders more often used a combination of stimulant and non-stimulant psychotropic medication compared to children with ADHD-only or ADHD and one disorder (37, 28, 19\%, respectively). Another study of Robison et al. examined the prevalence of single and combination treatment modalities for children aged 5-18 years with ADHD in relation to co-morbidity [25]. They reported that children with ADHD without co-morbidity were more likely to receive stimulant medication alone (51\%) and less likely to receive additional psychotherapy or mental health counselling (26\%) compared to all children with ADHD, with or without co-morbidity (42 and 32\%, respectively). Psychotropic co-medication was not taken into account in this study.

We conducted a survey among physicians of a nationwide sample of stimulant-treated children in the Netherlands, to investigate the use of psychosocial interventions and psychotropic co-medication among stimulant-treated children with ADHD in relation to the presence of psychiatric co-morbidity.

\section{Methods}

This research was a follow-up of a questionnaire survey among parents of stimulant-treated children in 2003. To obtain a nationwide community sample of stimulant-treated children irrespective of the prescribing physician, pharmacies were used for detecting records of stimulanttreated children in their pharmacy information system. Since all prescriptions from medical doctors are entered in the pharmacy information system, regardless of particular health insurance or reimbursement status of the medication, the pharmacy is a reliable source of detecting medication users [15].

In the first 3 weeks of May 2003, 115 pharmacies located all over the Netherlands identified current users of methylphenidate and dexamphetamine in their information system and sent a questionnaire to their carer. Current users were defined as children younger than 16 years for whom at least two stimulant prescriptions were dispensed between 1 November 2002 and 1 May 2003, of which at least one prescription was in 2003.

The findings of the parents' questionnaire and the response analysis were subject of another study and were published elsewhere $[8,9]$. For the present study, it was important that the parents' questionnaire included a request for an informed consent to approach the stimulant prescribing physician. Informed consent was needed from the parents, and also from children if aged 12 years and older. After informed consent was received, a questionnaire and a copy of the informed consent were sent to the physician currently prescribing stimulants for the child. The protocol was approved by the Medical Ethics Board of the University Medical Centre in Groningen.

\section{Questionnaire}

The questionnaire was tested among five physicians. The resulting questionnaire contained open-ended and multiple choice questions about stimulant treatment, psychiatric diagnoses, co-medication and several types of non-pharmacological psychosocial interventions focused at the child and/or the parents. Only psychosocial interventions delivered by specifically trained mental health professionals (e.g. psychologists, psychiatrists, psychotherapists, social workers) were included. Psychosocial interventions directly aimed at the child, such as individual or groupbased cognitive behavioural therapy, social skills and self-regulation training, were classified as child-focused 
interventions. Training which took a maximum of $2 \mathrm{~h}$ per week was classified as non-intensive, whereas intensive treatment programs took more than $2 \mathrm{~h}$ per week. Physiotherapy implied physical exercises for motor problems and all dietary interventions (e.g. elimination diet) were classified under "diet." Treatment for children admitted to a hospital or residential centre for mental disorders was defined as inpatient treatment. Treatment for children attending day hospital programs was defined as day treatment. Special education services were limited to children who attend specialized schools. Common and simple interventions at regular schools that do not require medical consultation, such as altered seating and home-schoolnotes were not qualified as psychosocial interventions in this study. Interventions aimed at parents to teach them specialized child-management techniques were classified as parent-focused interventions. Individual parent training or group-based parent training was distinguished. Home training is an intensive form of family training in the home setting, sometimes with the use of video recording. Other forms of therapy or courses for parents were classified as "other training/course."

\section{Analysis}

Questionnaires were regarded unsuitable for analysis if the questionnaires were returned blank or when the physician reported not being the prescriber. Children were classified based on their diagnosis as reported by their physician. Only children diagnosed with ADHD were included in the present study. When no other diagnosis was reported, the children were classified as "ADHD-only", when pervasive developmental disorder (autistic disorder or PDD-NOS) was reported as "ADHD + PDD", when one of these disruptive behaviour disorders was reported as "ADHD + ODD/CD", and when one or more psychiatric diagnoses other than PDD and ODD/CD were reported they were classified as "ADHD + other co-morbidity." Children with ADHD and both PDD and ODD/CD were excluded from the analysis.

We compared child characteristics and the use of psychosocial interventions and psychotropic co-medication in the four diagnostic groups. Omnibus tests were used to test whether the proportions in the four diagnostic groups differed significantly across each group. Significant Chisquare tests were followed by post hoc comparisons: (1) ADHD-only versus ADHD + PDD, (2) ADHD-only versus ADHD + ODD/CD, (3) ADHD-only versus ADHD + other co-morbidity, (4) ADHD + PDD versus ADHD + ODD/CD, (5) ADHD + PDD versus ADHD + other co-morbidity, (6) ADHD + ODD/CD versus ADHD + other co-morbidity. In general, a $P$-value $<0.05$ was considered statistically significant. To adjust for multiple comparisons, post hoc tests were considered significant at $P<0.01$. All analyses were performed using SPSS 11.0.

\section{Results}

The 115 pharmacies sent 1,307 questionnaires to parents of stimulant users. Of these, 924 questionnaires $(71 \%)$ were returned. In 811 of the 924 cases $(88 \%)$ permission was given to approach the physician, of which 773 contained a valid address. Of the 773 questionnaires sent to physicians, 556 questionnaires were returned $(72 \%)$. According to the physicians, 512 of the 556 children $(92 \%)$ were diagnosed with ADHD. Two subjects were diagnosed with ADHD and both PDD and ODD/CD, leaving 510 cases for the present study. Almost half of these questionnaires were filled out by general practitioners (GPs, 47\%). Child psychiatrists and paediatricians were responsible for, respectively, 27 and $23 \%$. The mean age of the stimulant-treated children with ADHD in this study was 10.5 years, and the male-to-female ratio was approximately 5.7:1.

\section{Co-morbidity}

Of the 510 stimulant-treated children with ADHD, the physician reported only the diagnosis of ADHD in 69\%. For the remaining children, the most frequently reported co-morbidities were PDD (53 children, 10.4\%), ODD/CD (50 children, 9.8\%), followed by learning disorder (28 children, 5.5\%), mental retardation (22 children, $4.3 \%$ ), tic disorder (10 children, $2.0 \%)$ and anxiety disorder (5 children, $1.0 \%$ ). Of the 53 children in the PDD group, 49 were diagnosed PDD-NOS and 4 with autistic disorder. Of the 50 children in the ADHD + ODD/CD group, 48 children were diagnosed with ODD and 5 with $\mathrm{CD} ; 3$ children were reported to have both ODD and CD. For 57 children (11\%) co-morbidity other than PDD or ODD/CD was reported.

\section{Type of prescriber}

The type of physician prescribing stimulants was associated with the presence of co-morbidity $(P<0.001)$. Of the children with ADHD and a co-morbid disorder, 35\% received their current stimulant prescriptions from a child psychiatrist, which was almost twice the percentage compared to children with ADHD-only. Stimulant prescriptions for children with ADHD-only were mostly provided by GPs. In $41 \%$ of the children with ADHD-only, stimulant prescribing was transferred from a child psychiatrist or paediatrician to a GP after initiation of treatment. For children with co-morbid PDD, ODD/CD or another psychiatric disorder this percentage was lower: 30, 18 and 23\%, respectively $(P<0.01$, data not shown). No relationship 
Table 1 Characteristics of stimulant-treated children diagnosed with ADHD-only, ADHD with PDD, ADHD with ODD/CD and ADHD with other psychiatric co-morbidity

\begin{tabular}{|c|c|c|c|c|c|}
\hline & \multirow{2}{*}{$\begin{array}{l}\text { ADHD-only } \\
n=350 \\
n\end{array}$} & \multicolumn{3}{|c|}{ ADHD + co-morbidity } & \multirow{2}{*}{$\begin{array}{l}\chi^{2} \text { test } \\
P\end{array}$} \\
\hline & & $\begin{array}{l}\text { ADHD }+ \text { PDD } \\
(n=53) \\
n\end{array}$ & $\begin{array}{l}\mathrm{ADHD}+\mathrm{ODD} / \mathrm{CD} \\
(n=50) \\
n\end{array}$ & $\begin{array}{l}\text { ADHD + other } \\
\text { co-morbidity }(n=57) \\
n\end{array}$ & \\
\hline Age (mean years) & $350(10.5)$ & $53(10.6)$ & $50(10.3)$ & $57(10.4)$ & 0.94 \\
\hline Male (\%) & $300(86)$ & $44(83)$ & $44(88)$ & $46(81)$ & 0.69 \\
\hline Specialized school (\%) & $98\left(28^{\mathrm{b}}\right)$ & $32\left(60^{\mathrm{a}}\right)$ & $20\left(40^{\mathrm{ab}}\right)$ & $25\left(44^{\mathrm{ab}}\right)$ & $<0.001$ \\
\hline \multicolumn{6}{|c|}{ Time since first prescription } \\
\hline$<1$ year $(\%)$ & $78(22)$ & $8(15)$ & $8(16)$ & $15(26)$ & \multirow[t]{4}{*}{0.42} \\
\hline $1-2$ years $(\%)$ & $64(18)$ & $10(19)$ & $14(28)$ & $12(21)$ & \\
\hline$>2$ years $(\%)$ & $203(58)$ & $33(62)$ & $26(52)$ & $30(53)$ & \\
\hline Unknown (\%) & $5(1)$ & $2(4)$ & $2(4)$ & $0(0)$ & \\
\hline \multicolumn{6}{|c|}{ Current prescriber stimulants } \\
\hline GP (\%) & $190\left(54^{\mathrm{a}}\right)$ & $18\left(34^{\mathrm{b}}\right)$ & $13\left(26^{\mathrm{b}}\right)$ & $15\left(26^{\mathrm{b}}\right)$ & \multirow[t]{5}{*}{$<0.001$} \\
\hline Paediatrician $(\%)$ & $79(23)$ & $13(25)$ & $8(16)$ & $18(32)$ & \\
\hline Child psychiatrist (\%) & $69(20)$ & $22(42)$ & $28(56)$ & $20(35)$ & \\
\hline Other $(\%)$ & $9(3)$ & $0(0)$ & $1(2)$ & $4(7)$ & \\
\hline Unknown (\%) & $3(1)$ & $0(0)$ & $0(0)$ & $0(0)$ & \\
\hline
\end{tabular}

Proportions that share the same superscript are not significantly different from each other

was found between the presence of co-morbidity and age, gender and time since the first dispensed stimulant prescription was filled $(P \geq 0.42)$.

Additional interventions

Overall, there is an association between the presence or absence of co-morbidity and attending a specialized school (Table 1). Sixty percent of the children with ADHD and co-morbid PDD were reported to attend a specialized school. This was twice as often compared to children with ADHD-only.

The presence or absence of co-morbidity was also associated with the use of psychosocial interventions aimed at the child (Table 2). Stimulant-treated children with ADHD and co-morbid PDD, ODD/CD or other psychiatric co-morbidity were reported to receive more psychosocial interventions aimed at the child than children with ADHD-only.

Children with ADHD and co-morbid PDD or co-morbid ODD/CD received intensive training, day treatment and treatment in an inpatient clinic more frequently than children with ADHD-only.

The presence of psychiatric co-morbidity was also associated with parent-focused interventions (Table 2). Parent-focused interventions were most frequently offered to parents of children with ADHD + PDD (89\%) or ODD/ CD (84\%). Home training was provided when a PDD diagnosis was present in almost a quarter of these families, almost three times more often compared to families of a child with ADHD-only. Of the children in the ADHD-only group, $26 \%$ did not receive any psychosocial interventions focused at the child or parents (data not shown). This percentage was only 18 in the ADHD + other co-morbidity group, 10 in the ADHD + ODD/CD group and 8 in the ADHD + PDD group.

Psychotropic co-medication

There is an association between presence or absence of co-morbidity and the use of psychotropic co-medication (Table 2). Use of psychotropic co-medication was most prevalent among stimulant-treated children with ADHD + PDD (36\%), which was significantly more frequent compared to children in the ADHD-only group (18\%). Antipsychotic use was the most prevalent co-medication among stimulant-treated children with ADHD + PDD (17\%). This was almost sixfold higher than in the ADHD-only group (3\%).

The frequency of melatonin use did not differ significantly between the groups. Clonidine, antiepileptics, antidepressants, and hypnotics/anxiolytics were not frequently used as co-medication.

\section{Discussion}

This study among showed that stimulant-treated children with ADHD and co-morbid psychiatric disorders received 
Table 2 Characteristics of additional interventions and psychotropic co-medication among stimulant-treated children diagnosed with ADHDonly, ADHD with PDD, ADHD with ODD/CD and ADHD with other psychiatric co-morbidity

\begin{tabular}{|c|c|c|c|c|c|c|c|c|c|}
\hline & \multirow{2}{*}{\multicolumn{2}{|c|}{$\frac{\text { ADHD-only }}{n=350}$}} & \multicolumn{6}{|c|}{ ADHD + co-morbidity } & \multirow{3}{*}{$\begin{array}{l}\chi^{2} \text { test } \\
P\end{array}$} \\
\hline & & & \multicolumn{2}{|c|}{$\mathrm{ADHD}+\mathrm{PDD}(n=53)$} & \multicolumn{2}{|c|}{$\mathrm{ADHD}+\mathrm{ODD} / \mathrm{CD}(n=50)$} & \multicolumn{2}{|c|}{$\begin{array}{l}\text { ADHD }+ \text { other } \\
\text { co-morbidity }(n=57)\end{array}$} & \\
\hline & $n$ & $\%$ & $n$ & $\%$ & $n$ & $\%$ & $n$ & $\%$ & \\
\hline \multicolumn{10}{|l|}{ Psychosocial interventions } \\
\hline Child-focused interventions-any & 189 & $54^{\mathrm{b}}$ & 41 & $77^{\mathrm{a}}$ & 37 & $74^{\mathrm{a}}$ & 42 & $74^{\mathrm{a}}$ & $<0.001$ \\
\hline Non-intensive training & 108 & 31 & 13 & 25 & 17 & 34 & 18 & 32 & 0.27 \\
\hline Physiotherapy & 62 & 18 & 16 & 30 & 10 & 20 & 18 & 32 & 0.12 \\
\hline Diet & 15 & 4 & 6 & 11 & 1 & 2 & 1 & 2 & - \\
\hline Intensive training & 27 & $8^{\mathrm{b}}$ & 17 & $32^{\mathrm{a}}$ & 14 & $28^{\mathrm{a}}$ & 4 & $7^{\mathrm{b}}$ & $<0.001$ \\
\hline Day treatment & 22 & $6^{\mathrm{b}}$ & 16 & $30^{\mathrm{a}}$ & 11 & $22^{\mathrm{a}}$ & 7 & $12^{\mathrm{ab}}$ & $<0.001$ \\
\hline Inpatient treatment & 11 & $3^{\mathrm{b}}$ & 9 & $17^{\mathrm{a}}$ & 11 & $22^{\mathrm{a}}$ & 3 & $5^{\mathrm{ab}}$ & $<0.001$ \\
\hline Parent-focused interventions-any & 223 & $64^{\mathrm{c}}$ & 47 & $89^{\mathrm{a}}$ & 42 & $84^{\mathrm{ab}}$ & 36 & $63^{\mathrm{bc}}$ & $<0.001$ \\
\hline Individual parent training & 193 & $55^{\mathrm{b}}$ & 41 & $77^{\mathrm{a}}$ & 40 & $80^{\mathrm{a}}$ & 34 & $60^{\mathrm{ab}}$ & $<0.01$ \\
\hline Group based parent training & 29 & 8 & 9 & 17 & 7 & 14 & 1 & 2 & - \\
\hline Home training & 28 & $8^{\mathrm{b}}$ & 12 & $23^{\mathrm{a}}$ & 5 & $10^{\mathrm{ab}}$ & 4 & $7^{\mathrm{ab}}$ & $<0.01$ \\
\hline Other training/course & 26 & 7 & 5 & 9 & 6 & 12 & 8 & 14 & 0.10 \\
\hline \multicolumn{10}{|l|}{ Psychotropic co-medication } \\
\hline Any & 63 & $18^{\mathrm{b}}$ & 19 & $36^{\mathrm{a}}$ & 14 & $28^{\mathrm{ab}}$ & 10 & $18^{\mathrm{ab}}$ & 0.012 \\
\hline Melatonin & 41 & 12 & 7 & 13 & 8 & 16 & 4 & 7 & 0.49 \\
\hline Antipsychotics & 11 & $3^{\mathrm{b}}$ & 9 & $17^{\mathrm{a}}$ & 5 & $10^{\mathrm{ab}}$ & 5 & $9^{a b}$ & $<0.001$ \\
\hline Clonidine & 7 & 2 & 2 & 4 & 0 & 0 & 0 & 0 & - \\
\hline Antiepileptics & 3 & 1 & 2 & 4 & 1 & 2 & 1 & 2 & - \\
\hline Antidepressants & 1 & 0 & 1 & 2 & 0 & 0 & 0 & 0 & - \\
\hline Hypnotics/anxiolytics & 1 & 0 & 0 & 0 & 0 & 0 & 0 & 0 & - \\
\hline
\end{tabular}

Proportions that share the same superscript are not significantly different from each other

- Denotes Chi-square not valid due to small numbers

more additional psychosocial interventions and co-medication than children with an ADHD diagnosis only. The type of psychotropic co-medication and interventions received depended on the type of co-morbid disorder.

\section{Co-morbidity}

For $31 \%$ of cases psychiatric co-morbidity was reported in this sample of stimulant-treated children with ADHD. Other studies reported higher co-morbidity percentages ranging from $44 \%$ in community-derived samples to $87 \%$ in clinically-referred children diagnosed with ADHD [18]. In particular, the presence of ODD/CD in our study (10\%) was very low compared to, e.g. the 50-60\% reported by Gillberg [10]. Our low co-morbidity figures may be explained by a number of phenomena. First, low co-morbidity figures may be explained by under-diagnosis of psychiatric disorders because not all children were thoroughly screened by child psychiatrists. Second, may be physicians were not inclined to report diagnoses that do not support the prescription of stimulants since stimulant use was the entry point for this study. In particular, when the current prescriber was a GP it is likely that the first prescriber was a specialist. When the communication between the GP and specialist was less than ideal, the patient's medical record in the GPs documentation could be incomplete.

Pervasive developmental disorder as the most frequently mentioned co-morbid disorder, warrants special attention. Within the DSM-IV classification system, ADHD and PDD are exclusionary diagnoses, and therefore it is impossible to give a PDD and an ADHD classification to the same patient. There has been increasing debate about this issue $[6,11,16,23,31]$. The general point of the critics of the exclusionary criterion is that the overlap in clinical symptoms between ADHD and PDD is evident in practice, and removing the exclusionary criteria may be beneficial for stimulating research and optimizing treatment in these children [16, 23, 31]. It seems that many clinicians in the Netherlands have dropped the DSM-IV exclusionary criterion in clinical practice. 
Type of prescriber

In the Netherlands, a GP often takes over responsibility for prescribing medication which was initiated by a specialist. We found this was most frequently true for prescribing stimulant medication for children with ADHD-only. However, the majority of the children with psychiatric co-morbidity still received their stimulant prescriptions from a specialist, even in case of children who had been using stimulants for over 2 years. This difference may be explained by the fact that in more complicated cases the specialist remains closely involved with the child's treatment after its initiation.

\section{Additional interventions}

In this study, stimulant-treated children with ADHD and co-morbidity and their parents received considerably more treatment modalities than children with ADHD-only. Similar findings have been reported on the basis of US studies [4, 21]. Most guidelines recommend the combination of pharmacological and psychosocial interventions [2, $3,14,29]$. This is supported by evidence from the MTA study, reporting a somewhat higher efficacy of multimodal interventions in case of ADHD and co-morbid disorders $[13,30]$. However, a substantial proportion of the children in our study did not receive additional interventions. It may be that many children with ADHD without co-morbidity and even some children with co-morbidity respond adequately to pharmacological treatment only. However, it is not unlikely that some children receive less than optimal care. This is aggravated by our finding that almost $20 \%$ of the stimulant-treated youths were not monitored [9], so in those cases the treatment outcome is unknown to the clinician.

\section{Psychotropic co-medication}

We found an association between the presence of psychiatric co-morbidity and the use of psychotropic co-medication among stimulant-treated children with ADHD. This is in line with the few earlier reports from the USA [4, 21]. However, Radigan et al. reported an increased use of alpha agonists, antipsychotics, antidepressants, antiepileptics and anxiolytics among Medicaid children with ADHD and a co-morbid psychiatric disorder [21], while we found only an increased use of antipsychotics among children with ADHD and co-morbid PDD. Part of this difference may be explained by different prescribing practices in the USA and the Netherlands.

The more frequent use of antipsychotics among children with ADHD and PDD may be understood as confirmation that the presence of PDD symptoms in children with
ADHD has practical implications for treatment. After all, several studies have reported a positive effect of antipsychotics in reducing aggressive behaviour in children with PDD and disruptive behaviour disorders [5, 7, 24]. In contrast to reports from the USA, we found antipsychotics to be the most prevalent co-medication instead of antidepressants. In our study, co-medication with antidepressants was reported in less than $2 \%$, compared to $4-7 \%$ in the USA [17, 20, 32]. This lower use of anti-depressants may be partly explained by differences in timing of the study periods, but is probably more likely to be explained by cross cultural differences in medical practice, including the attention paid to internalizing versus externalizing disorders.

\section{Limitations}

Children with ADHD who did not receive stimulants were not included in our study and therefore the findings of our study cannot be generalized to all children with ADHD. Another limitation of our study is that no information was available on how children were assessed, so no statements could be made about the appropriateness or the relative weight of the diagnoses.

Our two-step recruitment procedure implied that physicians could only be approached if the responding parents and their child gave their consent, which may have led to selection bias. No association was found between giving consent and the child's age, gender or the type of prescribing physician, but physician response rate differed significantly for the type of prescribing physician, being highest for paediatricians (83\%) and lowest for child psychiatrists $(62 \%)$ [8].

Physicians were the only information source in this study. This may possibly have resulted in an underestimation of psychosocial interventions, because physicians may not be aware of all interventions in non-medical settings, for example at school. However, in the Netherlands mental health interventions are not delivered through the school system like in the USA. Individual children can receive didactic interventions such as remedial teaching within the school system, but individualized behaviour management programs are not available on regular schools or are always part of an elaborate behaviour management program put in place through health care professionals, with teachers as "co therapists." Using the current prescriber of stimulant medication as the only information source also may have lead to information bias. GPs taking over prescribing responsibility may have less information and knowledge about psychosocial interventions than psychiatrists or paediatricians. This may have resulted in an underestimation of interventions mentioned by GPs. 


\section{Conclusion}

Multimodal treatment of youth with ADHD is a common medical practice in the Netherlands, particularly for youth with co-morbid psychiatric disorders. Whether this reflects what is known about the effectiveness of multimodal interventions for children with ADHD and co-morbidity and their parents is unknown and might be subject for further research. The use of psychotropic co-medication for youth has led to some concerns, specifically about the safety of combining stimulants with antipsychotics for children diagnosed with ADHD and PDD.

Acknowledgments This study was funded by the Dutch National Health Insurance Board. Financial disclosures: Luuk Kalverdijk, Hilde Tobi and Adrianne Faber received a limited grant from JanssenCilag. Ruud Minderaa received a limited grant from Eli Lilly and is an advisor in the field of ADHD for Janssen-Cilag and Eli Lilly in Europe.

Open Access This article is distributed under the terms of the Creative Commons Attribution Noncommercial License which permits any noncommercial use, distribution, and reproduction in any medium, provided the original author(s) and source are credited.

\section{References}

1. Accardo P, Blondis TA (2001) What's all the fuss about Ritalin? J Pediatr 138:6-9

2. American Academy of Child and Adolescent Psychiatry (1997) Practice parameters for the assessment and treatment of children, adolescents, and adults with attention-deficit/hyperactivity disorder. J Am Acad Child Adolesc Psychiatry 36:85S-121S

3. American Academy of Pediatrics (2001) Clinical practice guideline: diagnosis an evaluation of the child with attentiondeficit hyperactivity disorder. Pediatrics 108:1033-1044

4. Boles M, Lynch FL, DeBar LL (2001) Variations in pharmacotherapy for attention deficit hyperactivity disorder in managed care. J Child Adolesc Psychopharmacol 11:43-52

5. Buitelaar JK, van der Gaag RJ, Cohen-Kettenis P et al (2001) A randomized controlled trial of risperidone in the treatment of aggression in hospitalized adolescents with subaverage cognitive abilities. J Clin Psychiatry 62:239-248

6. Clark T, Feehan C, Tinline C et al (1999) Autistic symptoms in children with attention deficit-hyperactivity disorder. Eur Child Adolesc Psychiatry 8:50-55

7. Delbello M, Grcevich S (2004) Phenomenology and epidemiology of childhood psychiatric disorders that may necessitate treatment with atypical antipsychotics. J Clin Psychiatry 65(Suppl 6):12-19

8. Faber A, van den Berg LTW, Netjes KA et al (2005) Recruitment of parents and physicians of stimulant-using children via community pharmacies is successful. J Clin Epidemiol 58:1072-1073

9. Faber A, Kalverdijk LJ, Hugtenburg JG et al (2006) Parents report on stimulant-treated youths in the Netherlands: initiation and follow-up care. J Child Adolesc Psychopharmacol 16:432440

10. Gillberg C, Gillberg IC, Rasmussen P et al (2004) Co-existing disorders in ADHD-implications for diagnosis and intervention. Eur Child Adolesc Psychiatry 13:I80-I92
11. Goldstein S, Schwebach AJ (2004) The co-morbidity of pervasive developmental disorder and attention deficit hyperactivity disorder: results of a retrospective chart review. J Autism Dev Disord 34:329-339

12. Hoagwood K, Kelleher KJ, Feil M et al (2000) Treatment services for children with ADHD: a national perspective. J Am Acad Child Adolesc Psychiatry 39:198-206

13. Jensen PS, Hinshaw SP, Kraemer HC (2001) ADHD co-morbidity findings from the MTA study: Comparing co-morbid subgroups. J Am Acad Child Adolesc Psychiatry 40:147-158

14. Kutcher S, Aman M, Brooks SJ et al (2004) International consensus statement on attention-deficit/hyperactivity disorder (ADHD) and disruptive disorders (DBDs): clinical implications and treatment practice suggestions. Eur Neuropsychopharmacol 14:11-28

15. Leufkens HGM, Urquhart J (2000) Automated pharmacy record linkage in the Netherlands. In: Strom BL (ed) Pharmacoepidemiology, 3rd edn. Wiley, Chichester

16. Luteijn EF, Serra M, Jackson S et al (2000) How unspecified are disorders of children with a pervasive developmental disorder not otherwise specified? A study of social problems in children with PDD-NOS and ADHD. Eur Child Adolesc Psychiatry 9:168-179

17. Martin A, Van Hoof T, Stubbe D et al (2003) Multiple psychotropic pharmacotherapy among child and adolescent enrollees in Connecticut Medicaid managed care. Psychiatr Serv 54:72-77

18. Mash EJ, Barkley RA (2003) Child pathology. The Guilford Press, New York

19. Miller AR, Lalonde CE, McGrail KM et al (2001) Prescription of methylphenidate to children and youth, 1990-1996. Can Med Assoc J 165:1489-1494

20. Olfson M, Marcus SC, Weissman MM et al (2002) National trends in the use of psychotropic medications by children. J Am Acad Child Adolesc Psychiatry 41:514-521

21. Radigan M, Lannon P, Roohan P et al (2005) Medication patterns for attention-deficit/hyperactivity disorder and co-morbid psychiatric conditions in a low-income population. J Child Adolesc Psychopharmacol 15:44-56

22. Reid R, Hakendorf P, Prosser B (2002) Use of psychostimulant medication for ADHD in South Australia. J Am Acad Child Adolesc Psychiatry 41:906-913

23. Research Units on Pediatric Psychopharmacology Autism Network (2005) Randomized, controlled, crossover trial of methylphenidate in pervasive developmental disorders with hyperactivity. Arch Gen Psychiatry 63:1266-1274

24. Research Units on Pediatric Psychopharmacology Autism Network (2001) Risperidone in children with autism and serious behavioral problems. N Engl J Med 347(5):314-321

25. Robison LM, Sclar DA, Skaer TL et al (2004) Treatment modalities among US children diagnosed with attention-deficit hyperactivity disorder:1995-99. Int Clin Psychopharmacol 19:17-22

26. Safer DJ (2000) Are stimulants overprescribed for youths with ADHD? Ann Clin Psychiatry 12:55-62

27. Sawyer MG, Rey JM, Graetz BW et al (2002) Use of medication by young people with attention-deficit/hyperactivity disorder. Med J Australia 177:21-25

28. Schirm E, Tobi H, Zito JM et al (2001) Psychotropic medication in children: a study from the Netherlands. Pediatrics 108:e25

29. Taylor E, Döpfner M, Sergeant J et al (2004) European clinical guidelines for hyperkinetic disorder-first upgrade. Eur Child Adolesc Psychiatry 13:7-30

30. The MTA Cooperative Group (1999) A 14-month randomized clinical trial of treatment strategies for attention-deficit/hyperactivity disorder. Arch Gen Psychiatry 56:1073-1086

31. Yoshida Y, Uchiyama $T$ (2004) The clinical necessity for assessing attention deficit/hyperactivity disorder (AD/HD) 
symptoms in children with high-functioning pervasive developmental disorder (PDD). Eur Child Adolesc Psychiatry 13:307314

32. Zito JM, Safer DJ, DosReis S et al (1999) Psychotropic medication patterns for youths with attention deficit hyperactivity disorder. Arch Pediatr Adolesc Med 153:1257-1263
33. Zito JM, Safer DJ, DosReis S et al (2000) Trends in the prescribing of psychotropic medications to preschoolers. JAMA 283:1025-1030 